\title{
A Pilot Digital Image Processing Approach for Detecting Vineyard Parcels in Douro Region through High- Resolution Aerial Imagery
}

\author{
Telmo Adão \\ INESC TEC (formerly INESC \\ Porto) and \\ University of Trás-os-Montes e \\ Alto Douro \\ Vila Real, Portugal \\ telmoadao@utad.pt
Pedro Marques
University of Trás-os-Montes e
Alto Douro
School of Science and
Technology
Vila Real, Portugal
pedro.marques@utad.pt

\author{
Luís Pádua \\ University of Trás-os-Montes e \\ Alto Douro \\ School of Science and \\ Technology \\ Vila Real, Portugal \\ luispadua@utad.pt \\ Emanuel Peres \\ INESC TEC (formerly INESC \\ Porto) and \\ University of Trás-os-Montes e \\ Alto Douro \\ Vila Real, Portugal \\ eperes@utad.pt
}

\begin{abstract}
Vineyard parcels delimitation is a preliminary but important task to support zoning activities, which can be burdensome and timeconsuming when manually performed. In spite of being desirable to overcome such issue, the implementation of a semi-/fully automatic delimitation approach can meet serious development challenges when dealing with vineyards like the ones that prevail in Douro Region (north-east of Portugal), mainly due to the great diversity of parcel/row formats and several factors that can hamper detection as, for example, interrupted rows and inter-row vegetation. Thereby, with the aim of addressing vineyard parcels detection and delimitation in Douro Region, a preliminary method based on segmentation and morphological operations upon highresolution aerial imagery is proposed. This method was tested in a data set collected from vineyards located at the University of Tr'as-os-Montes and Alto Douro(Vila Real, Portugal). The presence of some of the previously mentioned challenging conditions - namely interrupted rows and inter-row grassing - in a few parcels contributed to lower the overall detection accuracy, pointing out the need for future improvements. Notwithstanding, encouraging preliminary results were achieved.
\end{abstract}

Permission to make digital or hard copies of all or part of this work for personal or classroom use is granted without fee provided that copies are not made or distributed for profit or commercial advantage and that copies bear this notice and the full citation on the first page. Copyrights for components of this work owned by others than ACM must be honored. Abstracting with credit is permitted. To copy otherwise, or republish, to post on servers or to redistribute to lists, requires prior specific permission and/or a fee. Request permissions from Permissions@acm.org.

ICGDA '18, April 20-22, 2018, Prague, Czech Republic

(C) 2018 Association for Computing Machinery.

ACM ISBN 978-1-4503-6445-4/18/04 ..\$15.00

DOI: https://doi.org/10.1145/3220228.3220237

\section{CCS Concepts}

- Applied computing $\rightarrow \quad$ Agriculture;

^ Computingmethodologies $\rightarrow \quad$ Image processing;

$\wedge$ Information sys-tems $\rightarrow$ Geographic information systems.

\section{Keywords}

Vineyard parcel; Zoning; Automatic Vine Parcelling; DigitalImage Processing; UAV; UAS; RGB

\section{INTRODUCTION}

Vine parcelling towards viticultural zoning constitutes the preliminary step for significantly important activities such as propriety assignment, cultures characterization and landuse management and planning. However, in the literature concerning automatic detection of vineyard elements, works focusing vine rows processing seem to be more frequent than the ones addressing vineyard parcelling.

Regarding the works associated to vineyard rows detection, Bobillet et al. [1] proposed a method based on active contour model. Comba et al. [2] combined dynamic segmentation, Hough Space Clustering and Total Least Squares techniques with good results, even in the presence of disturbance elements (inter-row grassing, shadows, etc.). Same techniques were applied by Primicerio et al. [3] in multispectral images processed with normalized difference vegetation index (NDVI). Later, Primicerio et al. [4] went further and applied a multi-logistic model for the detection of missing plants in vine rows. Nolan et al. [5] described a way of detecting vine rows with skeletonisation techniques. Other works similarly aligned with this context can be found using different strategies such as row detection involving Ward's modified technique [6], vine/non-vine discrimination through crop surface models production [7], performance comparison between classification approachesfor vines canopy estimation on vertical trellis systems [8] andvineyard rows characterization based on dense point cloud[9]. 
On the other hand, semi-/fully automatic vine parceling approaches seems to be harder to find. Delenne et al. [10] reported a methodology focusing vineyards delineation in Languedoc-Roussillon region (southern France), based on their previous methods using Fast Fourier Transform (FFT) $[11,12]$ and image subsets with recursive segmentation $[13,14]$. Another work was presented by Adam et al. [15] who combined lidar data with a focal statistics method. Karakiziand Karantzalos [16] developed an object-based detection and discrimination framework operating with very high resolution pan-sharpened satellite data, which was validated on four different viticulture regions in Greece. Regarding vine parcels detection, a standard nearest neighbour classifier was adopted. Recently, a machine learning-based approach combining superpixels and supervised classification was proposed [17], also using satellite imagery.

Acknowledging vine parcelling importance, the aforementioned scarcity of approaches addressing this topic and, also, the numerous vineyard morphologies that can be found in Douro's demarcated region constitute the motivation for this paper, in which an alternative method for detecting vine parcels out of high-resolution aerial imagery is presented. This method is still in an early development stage, but promising preliminary results were achieved by processing images collected with an unmanned aerial vehicle (UAV), whose flights were carried out over vineyard fields located at the University of Tr'as-os-Montes and Alto Douro (UTAD- Vila Real, Portugal).

Regarding paper's organization, next section will provide some details regarding the images that were collected and used for further processing. Section 3 describes the method used for processing UAV collected images with the goal of detecting vine parcels. Some results are provided in Section4, just before the final one (Section 5) that is reserved for conclusions and remarks announcing future developments.

\section{AERIAL IMAGERY CHARACTERIZA- TION}

Douro Region has a wide variety of vineyard formats, with many delineations and several frontiers with each others. Their rows can be rectilinear or curvilinear and each one of them can be simply or pair-wisely arranged. Moreover, it is quite common to find several vine parcels with missing vine plants within the same row and/or inter-row vegetation connecting two parallel rows within the same vine parcel. All of this heterogeneity turns out to be a convenient case study to exploit digital approaches for automatic features extraction.

Considering the early stage of this study in particular, a simpler imagery data set collected at UTAD campus, more specifically in

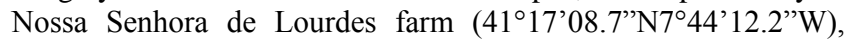
was used. This area is mainly composed of vineyard parcels besides other much less predominant crops such as maize, a hazelnut orchard, olive trees and other fruit trees - and its surface height is practically homogeneous. At acquisition moment, some of those parcels had interference of inter-row vegetation and/or interrupted rows. Figure1 depicts the imagery used in this study, with a semi- transparent yellow layer highlighting the targeted vineyards. A fixed-wing UAV senseFly eBee (senseFly SA, Loussagne, Switzerland) was used to carry out the data acquisition missions. Flight planning operations were configured using eMotion software. Flight height and overlap were respectively set

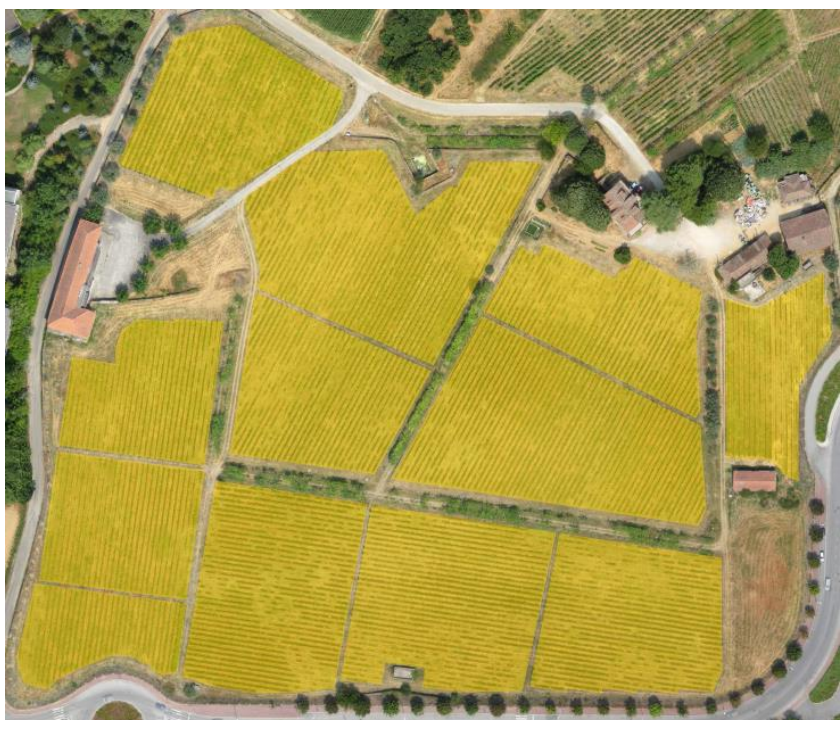

Figure 1: Study area, located at Nossa Senhora deLourdes farm $\left(41^{\circ} 17^{\prime} 08.7^{\prime} \mathrm{N} 7^{\circ} 44^{\prime} 12.2^{\prime}\right.$ ' $\left.\mathrm{W}\right)$, nearbythe campus of the University of Tr'as-os-Montes eAlto Douro. Semi-transparent yellow layer high-lights the targeted vineyards.

up to $175 \mathrm{~m}$ and $75 \%$, while area-related parameters were defined to reach $\approx 12$ ha of land covering. A resulting ground sample distance (GSD) of $\approx 5 \mathrm{~cm}$ was obtained. To perform the RGB imagery acquisition during the mission, a Canon IXUS $127 \mathrm{HS}$ camera was placed on-board of the UAV.

Acquired data photogrammetric processing was carried out in Pix4Dmapper Pro (Pix4D, Lousagne, Switzeland).This software is capable of dealing with different electromagnetic spectrum imagery and their potential distortions. To perform photogrammetric processing, it was used a laptop with the following characteristics:

- $\quad$ an Intel i7-4720HQ central processing unit, running at 2.6 $\mathrm{GHz}$;

- $\quad 16 \mathrm{~GB}$ of Random Access Memory (DDR3, $1600 \mathrm{MHz}$ );

- NVidia GeForce GTX 970m (3GB GDDR5 5000 MHz) graphic card.

Acquired RGB imagery was imported to Pix4D and a traditional photogrammetric processing pipeline was applied: firstly, dense point clouds were created and, then, the orthophotomosaics were obtained, followed by digital surface models.

Next section will present the method proposed in this paper, which accepts corrected orthophoto mosaics as input and manages to find vineyard parcels by automatically processing the features present in the image.

\section{METHOD PROPOSAL}

At this development stage, the proposed method for vineyard parcelling works with four main groups of steps, as itis portrayed by Figure 2: basic segmentation, region proprieties analysis, cleaning of loose pixels and division of objects into groups of orientation and, lastly, vine parcels handing. Matlab (MathWorks Inc, Massachusetts, United States of America) has been the supporting tool for implementation. After image loading, the first group of operations carries out color space manipulation in which 
RGB image is converted Hue-Saturation-Value (HSV). Then, image greenness is filtered through band "H" to keep vegetation and dispose everything else (replaced with black color). Image is reconverted to RGB colour space, followed by an adaptive threshold application based on local information.

During the region proprieties analysis, objects are splitted by clusters of white pixels and the following features are extracted: region of interest image and bounding box (for object's isolated processing and further original position retrieval), area in pixels (towards small objects removal), centroid, orientation, major/minor axis length and eccentricity(allowing rough row characterization), as well as convex hull(for row contact testing).These objects are gathered in arrays of structures holding previously extracted features, which are then submitted to the next step: cleaning and grouping by orientation. Cleaning will delete the rows with small eccentricities, min axis lengths and areas. By analysing the objects of a $2604 \times 1613 p x$ image (downsampled from $8680 \times 5436 \mathrm{px}$ for processing burden decreasing purposes) with $5 \mathrm{~cm}$ of GSD, the following threshold values were established: 0.92 for excentricity_treshold ratio; 3 px for min_axis _treshold; and 15 px row_area_treshold.

The division of orientation groups intends to simplify the processing and decrease the probability of overlapping rows belonging to different vineyards. Thus, rows are grouped by $\pm \theta$ degrees. $\theta$ selected in this approach was $18^{\circ}$, which enables dealing with up to 20 different groups. Finally, vine parcels handling takes place. For each orientation group, an image is created and burned with their respective rows. Then, a dilation is performed to force neighbor rows to contact. Dilation has wellknown mathematical definition, as it is pointed out by equation 1 :

$$
A \oplus B=\left\{\mathrm{z} \mid(\hat{B})_{\mathrm{z}} \cap A \neq \varnothing\right\}
$$

where $B$ and $\hat{B}$ refer to a structuring element affecting a pixel set $\mathrm{z}$, reflectively. A perpendicular line - relatively to each row was the structuring element used to perform the described operation. 25 px was the scalar that came out from the 2604x1613 px (downsampled) image analysis, to maximize side-by-side rows contact preventing, simultaneously, unintended connections with rows having similar orientations in the neighbour groups.

After applying dilation to the whole groups set, rows are tested for contact - with a polygon intersection algorithm -assigning the touching ones to independent structures representing vineyard parcels. When rows aggregation task is over, delimiting polygon is calculated for each one of those parcels - based in extremity rows -, resulting in the respective estimated area. A clean-up is performed in the end to discard vineyards with less than 5 objects.

Proposed method was tested against ground-though to assess its precision in detecting vineyard parcels. Results are shown in the following section.

\section{PRELIMINARY RESULTS}

To preliminarily test the accuracy of the method, twelve vineyard parcels belonging to the study area that can be seen in Figure 1 were numbered from 1 to 12 and manually delineated to produce ground-truth masks. Concurrently, method's pipeline was used to process the image and estimate vineyard parcels, considering the same numbering plan. Percentages of overlap between ground- truth and estimated vineyard parcels were then assessed through Dicesimilarity coefficient. Table 1 presents the analytical results that are also visually depicted in Figure 3.

As it turns out, ground-truth and estimated vineyard parcels overlap range is between around 33 and $94 \%$. Such disparity can be explained with the presence of inter-row vegetation and with the absence of vine vegetation in many rows, both affecting the performance of the proposed method.

On the one hand, inter-row vegetation ends up to form bigger clusters after image dilation, which are discarded when the eccentricity test is performed, resulting in severe underestimations, as it can be observed in vineyard parcels 1 and 2 , in which only $33.63 \%$ and $64.46 \%$ of detection rate(respectively) was achieved.

On the other hand, the lack of vines' vegetation also leads to the method's performance decrease, as it occurs in those cases characterized by the presence of inter-row vegetation, but due to opposite reasons. More specifically, image dilation may fail its goal of bonding some neighbour vine rows due to fragmentation. Underestimation in such conditions can be observed in vineyard parcel 11, wherein the lack of vines' vegetation ended up to result in a detection rate of $\approx 78 \%$.

Another phenomenon deriving from situations involving inter-row vegetation and/or vine rows interruptions that may take place is sub-parcelling, i.e., an unintended and uncontrolled event characterized by the division of vineyard parcels into smaller parts. Typically, sub-parcelling occurs when image dilation and subsequent polygon intersection tests merge two or more groups of rows that should be part of the same vineyard parcel. For example, the proposed method splitted vineyard parcel 11 into 4 sub-parcels, mainly due to several vine vegetation interruptions that resulted in vine rows fragmentation.

Notwithstanding the aforementioned issues, an average accuracy of $76.48 \%$ was achieved for the tested data set.

\section{CONCLUSIONS AND FUTUREWORK}

In this paper, a preliminary method for automatically detect vineyard parcels based on segmentation and morphological operations - such as dilations - was presented. This work intended to constitute a starting point to address more complex cases regarding Douro's vineyards, which typically have different morphologies. Together with the lack of vines' vegetation as well as the presence of plants between rows, such features represent a real challenge for automatic image based processing approaches.

The proposed pipeline mainly relies in image segmentation, elliptical objects filtering through eccentricity and transversal axis length measurements, dilation operations and polygon intersection tests to find clusters of vine row portions constituting vineyard parcels. Method's results are encouraging in what regards to vineyards that have a complete set of rows and absent inter-row vegetation, but the opposite conditions reveal that more work needs to be done. Therefore, the possibility of mitigating current method's issues with convolutional neural networks (CNNs) is being considered for future applications. To that end, powerful tools - e.g. Tensor Flow [18] and Keras [19] - capable of streamlining machine-based training/prediction of vineyards with different morphologies and in diverse vegetative conditions are available. 

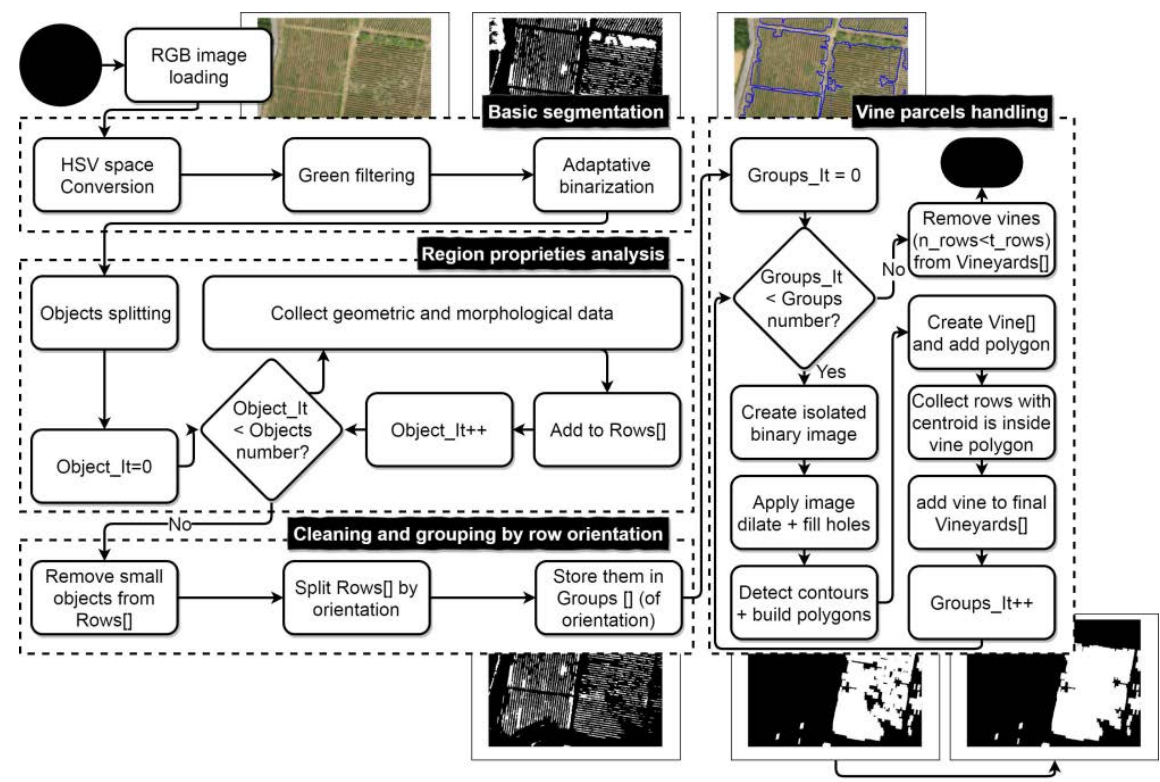

Figure 2: Proposed method flowchart, composed of four main groups of steps: basic segmentation, regionproprieties analysis, cleaning of loose pixels and division of objects into groups of orientation and, finally, vine parcels handing.

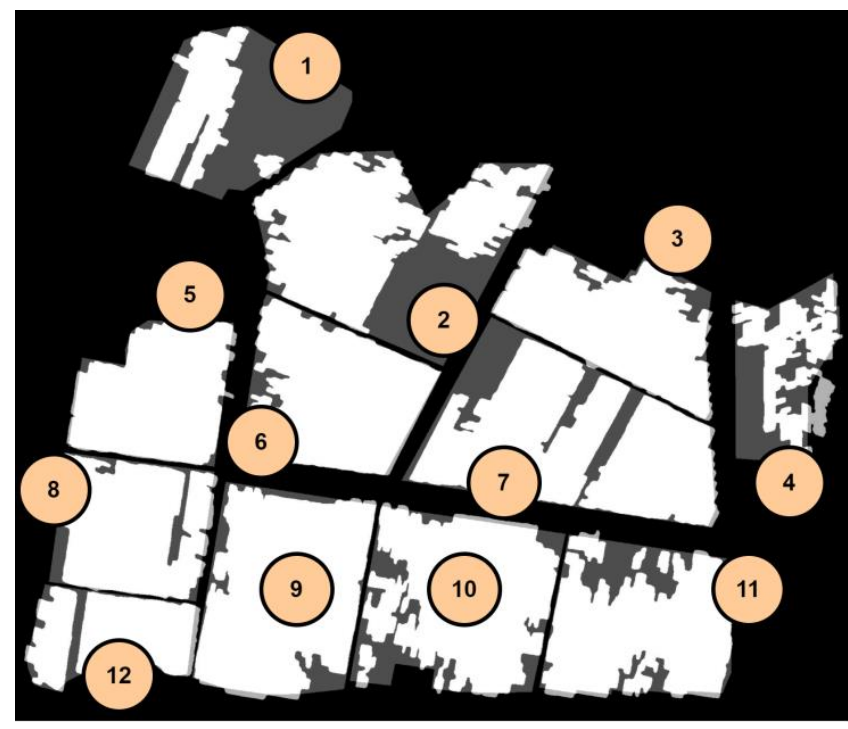

Figure 3: Binary mask - resulted from the applica-tion of the proposed method - overlapped to ground-truth mask, providing a visual insight of the meth-ods' accuracy.

\section{ACKNOWLEDGMENTS}

This work was financed by the European Regional Development Fund (ERDF) through the Operational Programme for Competitiveness and Internationalisation - COMPETE2020 under the PORTUGAL 2020 Partnership Agreement, and through the Portuguese National Innovation Agency(ANI) as a part of project "PARRA - Plataforma integrAda de monitoRização e avaliação da doença da flavescênciadou Rada na vinhA" (N³447) and ERDF and North 2020- North Regional Operational Program, as part of project "INNOVINE\&WINE - Vineyard and Wine Innovation Platform" (NORTE-01-0145-FEDER-000038).

\section{ADDITIONAL AUTHORS}

Additional authors: António Cunha, António M. R. de Sousa and Raul Morais; INESC TEC (formerly INESC Porto) and University of Trás-os-Montes e Alto Douro; \{acunha,amrs,rmorais\}@utad.pt. 


\begin{tabular}{|c|c|c|c|c|c|c|}
\hline ID & $\begin{array}{c}\text { Control } \\
\text { Area }\left(\mathrm{px}^{2}\right)\end{array}$ & $\begin{array}{c}\text { Control } \\
\text { Area (ha) }\end{array}$ & $\begin{array}{c}\text { Test Area } \\
\left(\mathrm{px}^{2}\right)\end{array}$ & $\begin{array}{c}\text { Test Area } \\
\text { (ha) }\end{array}$ & $\begin{array}{l}\text { Dice Sim. } \\
\text { Corr. }(\%)\end{array}$ & $\begin{array}{c}\text { Sub- } \\
\text { parcelling }\end{array}$ \\
\hline 1 & $1,384,891$ & 0.35 & 476,988 & 0.12 & 33.63 & 2 \\
\hline 2 & $2,196,294$ & 0.55 & $1,471,729$ & 0.37 & 64.46 & 2 \\
\hline 3 & $1,206,321$ & 0.30 & $1,123,091$ & 0.28 & 88.79 & 1 \\
\hline 4 & 818,283 & 0.20 & 482,040 & 0.12 & 46.61 & 1 \\
\hline 5 & $1,034,356$ & 0.26 & $1,022,438$ & 0.26 & 93.86 & 1 \\
\hline 6 & $1,330,266$ & 0.33 & $1,275,414$ & 0.32 & 92.49 & 1 \\
\hline 7 & $2,197,594$ & 0.55 & $1,798,897$ & 0.45 & 80.55 & 2 \\
\hline 8 & $1,106,894$ & 0.28 & 995,252 & 0.25 & 85.53 & 1 \\
\hline 9 & $1,733,591$ & 0.43 & $1,604,501$ & 0.40 & 89.61 & 1 \\
\hline 10 & $1,752,339$ & 0.44 & $1,464,909$ & 0.37 & 79.82 & 2 \\
\hline 11 & $1,474,603$ & 0.37 & $1,227,926$ & 0.31 & 78.47 & 4 \\
\hline 12 & 698,302 & 0.17 & 448,771 & 0.16 & 83.96 & 2 \\
\hline
\end{tabular}

Table 1: Accuracy results of the proposed method to detect vineyard parcels. Ground-truth and automaticallydetected vineyard parcels' areas are presented (in pixels and hectares) along with correlation percentages(calculated with Dice similarity coefficient) and sub-parcelling data (i.e., the number of parts in which a givenparcel is divided after the proposed method application).

\section{REFERENCES}

[1] W Bobillet, JP Da Costa, C Germain, O Lavialle, and G Grenier. Row detection in high resolution remote sensing images of vine fields. Precision Agriculture, page 81, 2003.

[2] Lorenzo Comba, Paolo Gay, Jacopo Primicerio, and Davide Ricauda Aimonino. Vineyard detection from unmanned aerial systems images. Computers and Electronics in Agriculture, 114:78-87, 2015.

[3] J Primicerio, P Gay, D Ricauda Aimonino, L Comba, A Matese, and SF di Gennaro. Ndvi-based vigour maps production using automatic detection of vine rows in ultra-high resolution aerial images. In Precision agriculture'15, pages 693-712. Wageningen Academic Publishers, 2015.

[4] Jacopo Primicerio, Giovanni Caruso, Lorenzo Comba, Alfonso Crisci, Paolo Gay, Silvia Guidoni, Lorenzo Genesio, Davide Ricauda Aimonino, and Francesco Primo Vaccari. Individual plant definitionand missing plant characterization in vineyards from high-resolution uav imagery. European Journal of Remote Sensing, 50(1):179-186, 2017.

[5] AP Nolan, S Park, S Fuentes, D Ryu, and H Chung. Automated detection and segmentation of vine rows using high resolution uas imagery in a commercial vineyard. In Proceedings of the 21st International Congress on Modelling and Simulation, Gold Coast, Australia, volume 29, pages 1406-1412, 2015.

[6] Nicola Puletti, Rita Perria, and Paolo Storchi. Unsupervised classification of very high remotely sensed images for grapevine rows detection. European Journal of Remote Sensing, 47(1):4554, 2014.

[7] S. Burgos, M. Mota, D. Noll, and B. Cannelle. Use of Very High-Resolution Airborne Images to Analyse 3d Canopy Architecture of a Vineyard. ISPRS -International Archives of the Photogrammetry, Remote Sensing and Spatial Information Sciences, pages 399-403, August 2015.

[8] Carlos Poblete-Echeverr'1a, Guillermo Federico Olmedo, Ben Ingram, and Matthew Bardeen. Detection and segmentation of vine canopy inultra-high spatial resolution rgb imagery obtained from unmanned aerial vehicle (uav): A case study in a commercial vineyard. Remote Sensing, 9(3), 2017.
[9] Marie Weiss and Fr'ed'eric Baret. Using 3d point cloudsderived from uav rgb imagery to describe vineyard 3dmacro-structure. Remote Sensing, 9 (2), 2017.

[10] Carole Delenne, Sylvie Durrieu, Gilles Rabatel, and Michel Deshayes. From pixel to vine parcel: A complete methodology for vineyard delineation and characterization using remote-sensing data. Computers and Electronics in Agriculture, 70(1):78 - 83, 2010.

[11] Carole Delenne. Extraction et caract'erisation desvignes 'a partir de donn'ees de t'el'ed'etection 'a tr'eshaute r'esolution spatiale: application enLanguedoc-Roussillon pour la constitution de bases dedonn'ees g'eographiques. $\mathrm{PhD}$ thesis, Ecole nationale dug'enie rural, des eaux et des for^ets (Paris; Nancy),2006.

[12] C Delenne, G Rabatel, V Agurto, and M Deshayes.Vine plot detection in aerial images using fourieranalysis. In $1 s t$ International Conference on Object-based Image Analysis (OBIA 2006), 2006.

[13] Gilles Rabatel, Carole Delenne, and Michel Deshayes. A non-supervised approach using gabor filters for vine-plot detection in aerial images. Computers and Electronics in Agriculture, 62(2):159 - 168, 2008.

[14] Carole Delenne, Gilles Rabatel, and Michel Deshayes. An automatized frequency analysis for vine plot detection and delineation in remote sensing. IEEE Geoscience and Remote Sensing Letters, 5(3):341-345, 2008.

[15] Adam J. Mathews and Jennifer L. R. Jensen. An airborne lidar-based methodology for vineyard parcel detection and delineation. International Journal of Remote Sensing, 33(16):5251-5267, 2012.

[16] C. Karakizi and K. Karantzalos. Detecting and classifying vine varieties from very high resolution multispectral data. In 2015 IEEE International Geoscience and Remote Sensing Symposium(IGARSS), pages 3401-3404, July 2015.

[17] A. Garc'ia-Pedrero, C. Gonzalo-Mart'in, and M. LilloSaavedra. A machine learning approach for agricultural parcel delineation through agglomerative segmentation. International Journal of Remote Sensing, 38(7):1809-1819, 2017.

[18] Google Brain. Tensorflow: An open-source software library for machine intelligence. https://www.tensorflow.org/.

[19] Fran, cois Chollet et al. Keras.

https://github.com/fchollet/keras, 2015. 